\title{
GAN TRANSISTOR IN SPACE EQUIPMENTS: FROM SELECTION TO APPLICATIONS
}

\author{
Philippe Fayt \& Marc Fossion, Paul Maynadier \& Michaël Notarianni
}

Thales Alenia Space Belgium \& France

Emails: firstname.name@thalesaleniaspace.com

\begin{abstract}
Two years ago, at ESPC-2014 results of ESA activity on $\mathrm{GaN}$ transistor survey has been presented. The main outcome was the existence of an unknown phenomena in silicon MOSFET: the dynamic Rdson.

Today we are the time of studying introduction of this technology into products. At least two suppliers (EPCco \& GaN Systems) are proposing stabilized GaN transistors components which are worth spending some time to try to implement in next generation product prototypes.
\end{abstract}

Space applications aren't very different from terrestrial ones when we speak about benefits of using GaN technology into dc-dc converters: lower losses \& more compact designs thanks to higher switching frequencies. Next to reliability analyses proposed by the component vendors to be able to address the automotive markets, there is of course quite a list "space" specific questions that need also to be answered to raise the confidence that $\mathrm{GaN}$ will one day be put is orbit: hermetic packaging \& assembly on PCBs, radiation harness, reliability impact of radiations, cooling in the absence of air flow, thermal cycling, drivers, deratings to apply...

In this article we presents some prototyping results \& shares some feedback \& concerns about the introduction of $\mathrm{GaN}$ into space qualified dc-dc products.

\section{CONTEXT}

GaN based transistors are promising for power applications because of their higher performances (low losses $\&$ high speed) and low size (from transistor size itself up to higher switching frequency ability)

Their introduction is progressive on other terrestrial applications, thanks to their progressive cost decrease. But their introduction for space application requests also at least :

- $\quad$ radiation hardness (both total dose and heavy ions)

- robustness \& reliability

- $\quad$ thermal draining (no convection)

\section{SELECTION}

A market survey of commercially available GaN transistors and diodes has been made by mid-2013, under ARTES 5.1 (Advanced Research in Telecommunications Systems) programme element funded by the European Space Agency, and entitled "Demonstration of High Power/High Efficiency Converter Using Commercially Available GaN" (contract 4F.067).

This survey is peculiar to a telecom application being a flight DC/DC converter, fed by the nowadays common $100 \mathrm{~V}$ satellite bus and with a power level of $600 \mathrm{~W}$. Small converters like POL (Point of Load) are clearly out of scope. This lead to make a survey on at least $200 \mathrm{~V}$ rated GaN components with a high current capability.

The contract encompasses the market survey and the procurement of the relevant parts [4], followed by a breadboard manufacturing using the best commercial GaN. The breadboard will be compared in performance with "classical" Si power MOSFET and diode within the selected DC/DC converter: a SMART topology i.e. a buck followed by a push-pull converter (see §5.1) further.

Even for GaN transistors having to working at $100 \mathrm{~V}$ bus or above, the Ron resistance becomes now comparable or better than $\mathrm{Si}$ MOSFET for power converters. However one important parameter has appeared in $\mathrm{GaN}$ HEMT components datasheets: dynamic Rdson. The phenomena seems now understood [6], measurements methods are now available. There is no more bad surprises \& we almost don't have to work with data sheets not mentioning dynamic Rdson. As a rough order of magnitude, one has to consider a factor x1.6 more conduction losses when using HEMT normally on devices in dc-dc switching conditions. Some experimental devices are even appearing where no dynamic Rdson are claimed [5].

Nevertheless, the interest of switching GaN is obvious thanks to their very low capacitances, allowing parallel operation without degrading significantly the switching losses (contrary to $\mathrm{Si}$ ). When the manufacturers will solve or at least improve the dynamic Ron performance, 
we will take full benefit of this promising component even at $100 \mathrm{~V}$ bus.

Another important point also linked to the $100 \mathrm{~V}$ bus voltage is the fact that the very well-known Figure of Merit (FOM) RDSon*QG is not relevant. For the targeted power converter of $600 \mathrm{~W}$, the QG losses represent only $4 \%$ of the switching losses against $75 \%$ due to Coss. We suggest for high power output and voltage above 50V a FOM = DynamicRDSon*Coss.

Finally, we see that within $2 . .3$ years packaging has strongly evolved. All GaN components using the good old TO-xx family of packages have passed over. Indeed high current transients requires low inductance packaging. Furthermore GaN HEMT transistors are now a 4 pin device as its almost mandatory to use Kelvin source to avoid parasitic turn-off.

For diodes, it seems that $\mathrm{SiC}$ devices have better performances than $\mathrm{GaN}$ in both forward voltage $\mathrm{Vd}(\mathrm{Id})$ and reverse current Ir. The main interest to work with those WBG diodes is their $\mathrm{dv} / \mathrm{dt}$ capability. Indeed, it is widely known that Si Schottky diodes have a limitation at $10 \mathrm{~V} / \mathrm{ns}$ whereas $\mathrm{GaN}$ and $\mathrm{SiC}$ have no such rating. Although $\mathrm{SiC}$ diodes are available their fail radiation testing. GaN diodes are still lagging behind in terms of performance. So if $\mathrm{GaN}$ transistors are to be used in classical buck or boost configurations, it is mandatory to resort to proceed to synchronous rectification.

For dc-dc applications, besides some specific operating conditions [7] dc-dc converters are hard to build using normally-on devices. For terrestrial applications, several component suppliers are moving forward in the cascade direction. For space applications this makes much less sense since a radiation hardened silicon MOSFET is needed to create the normally off feature on top of the $\mathrm{GaN}$ device which is used to manage high voltage.

The selection was done with the following criteria :

$$
\text { - Normally Off }
$$

- No leaded parts

When prospection was initiated mid 2013 were several parts coming from different manufacturers: EPC-co, Microsemi, GaN Systems, RFMD, Avogy, Transphorm, Panasonic, Fujitsu semiconductor, IR, MicroGaN, ... The world is changing extremely fast. There is no 3 months quarters happening without a new player coming in, some other disappearing \& other strategic alliances. The survey turned-out to be a permanent task to be stay up to date.

ESA itself has launched the development (project ESA A/O1-7688/13/NL/RA) of radhard components: "GaN devices for space based DCDC power conversion application» contract between ESA and OnSemi. Thales
Alenia Space is of involved in the project as end-user of these components.

While waiting for true radhard space qualified components, in order to proceed with investigations on GaN applicability into products, a pre-selection has to be made and Efficient Power Conversion Corp. \& Gan System Inc. are the candidates retained for current investigations in prototypes within Thales Alenia Space.

\section{EXPERIMENTAL RESULTS}

After component tests at parts level, implementation exercises started on several low, medium \& high applications.

\subsection{Multi-purpose low power dc-dc}

As part of the roadmap for product evolutions of the low power dc-dc, GaN transistors have been tested. In this type of low power applications, the use of $\mathrm{GaN}$ transistor could be viewed as completely overkill: current levels are significantly below the real capabilities of the transistors. Furthermore using advanced technology as $\mathrm{GaN}$ (which is still far away for being completely trusted) in a very low power allow to use it with considerable derating hence leading to a higher confidence in the reliability of the devices used in the context of this application. Such low power application also allow designers to get familiar with controlling, driving and electrically interconnecting GaN HEMT devices without having to take care on power dissipation aspects yet!

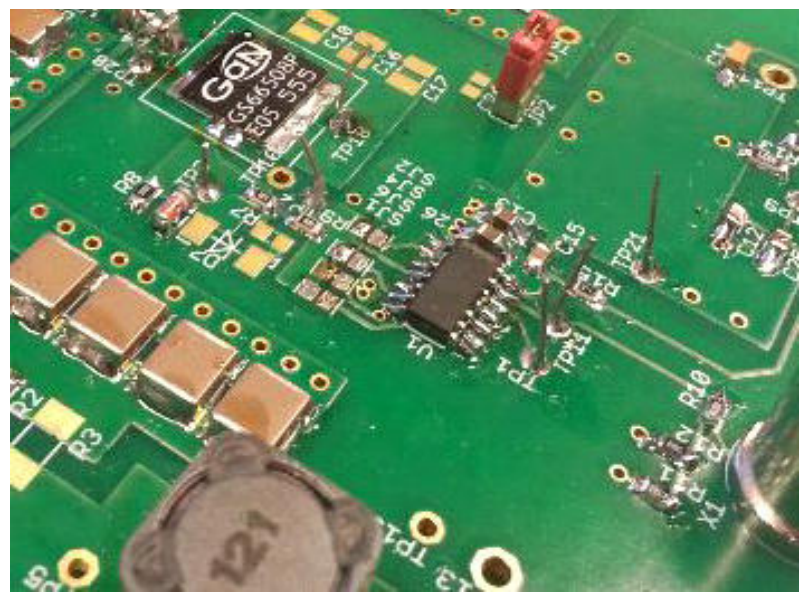

Figure 1. GaN-Systems devices under test

The interest of using GaN transistors in such low power equipment $(20 \ldots 40 \mathrm{~W})$ mainly lies in the availability of high voltage transistors capable of managing high input voltage ranges: one hand unregulated bus (22V to $37 \mathrm{~V})$ and on the other hand telecommunication satellite power bus at $100 \mathrm{~V}$. Today managing this large range requires two different hardware variants: one for high voltage with "high voltage" MOSFET transistors and another hardware variant for equipped with "low 
voltage" MOSFET. Using GaN allow to handle both voltages without compromising dc-dc efficiency. Next to that, as expected, size \& weight of the converter can be improved by using higher switching frequencies.

\subsection{EPC (Electrical Power Converter) for TWTA}

Electrical Power Conditioning unit for high voltage Travelling Wave Tube Amplifier is classified as a midrange power product with powers in the order of 200 to 600 Watts. They are present in satellite with rather high quantities: with 20 to 70 tubes per satellites. A recent trend in this product family in the assembly of so-called "dual EPC" where one module deliver high voltages for 2 TWTA resulting in a significant size, weight \& cost reduction at satellite level. For an equipment design perspective delivering twice more power from a unit is not that a simple tasks.

In the context of an ARTES 5.1 activity (see $\S 2$ ), GaN normally-on HEMT components have been tested as replacement parts for the usual silicon MOSFET transistors in the primary side of the dc-dc converter.

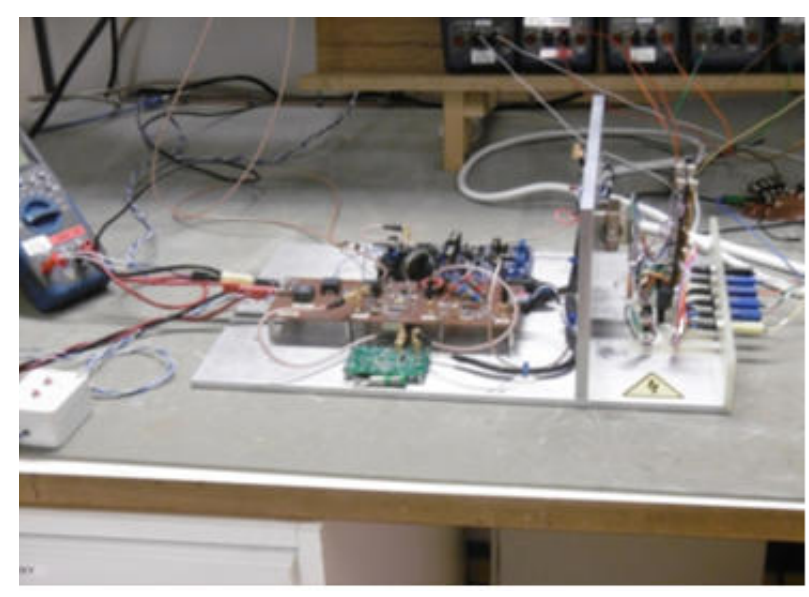

Figure 2. electrical prototype of $G a N d c-d c E P C$ (Electrical Power Converter) for TWTA

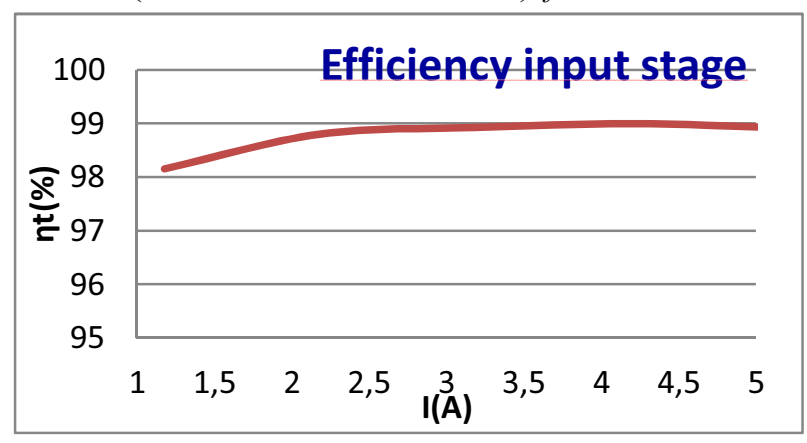

Figure 3. measured efficiency for $100 \mathrm{~V}$ input bus.

Efficiency of $99.0 \%$ at $250 \mathrm{KHz}$ achieved for the input stage. After several PCB layout iterations perfect turnon, turn-off waveform were achieved. The interest of using GaN lies (as usual) in the frequency increase: practically it means it is possible to transfer power level of dual EPC in a $\mathrm{HV}$ transformer of a single EPC at same efficiency.

Lessons learned from these prototypes are that thermal management of $\mathrm{GaN}$ is much more complex than with classical silicon MOSFET \& hence to be considered as a drawback of the GaN technology. Although GaN devices are dissipating much less than silicon MOSFET, there physical side being significantly lower makes the heat dissipation more complex. Not surprisingly also, the speed of this transistors request careful PCB layout to prevent oscillations, spikes $\&$ gate overstress.

\subsection{PPU}

Power supply system for electrical propulsion is the family of product where real high power are being considered with a target of $5 \mathrm{~kW}$ per module. Clearly in these type of products we are coming close to component ratings \& thermal challenges are very complex to manage. The introduction of GaN devices with their new packaging options (flip chip for Efficient Power Corp. \& the innovative packaging of GaNSystems) into high power space product will require significant efforts to create new mechanical structure \& assembly process to be able to evacuate high power dissipation without air flow while still managing high currents i.e. keeping low inductances everywhere.

\subsection{Low power RF units}

As described in [2], telecommunication receivers are widely used in geostationary satellites. On this low power application, the power supply delivers around $10 \mathrm{~W}$ through several output voltages, with strong requirements on output conducted ripple. The switching frequency has been set at $1 \mathrm{MHz}$ and the switching speed of the device was up to $30 \mathrm{~V} / \mathrm{ns}$. The prototype was $50 \%$ smaller but owing an additional tool, it was integrated in a RF unit. The RF output clearly "saw" the new switching frequency and additional simple LC filter suppress it. The prototype worked 1000 hours at $25^{\circ} \mathrm{C}$ without failure.

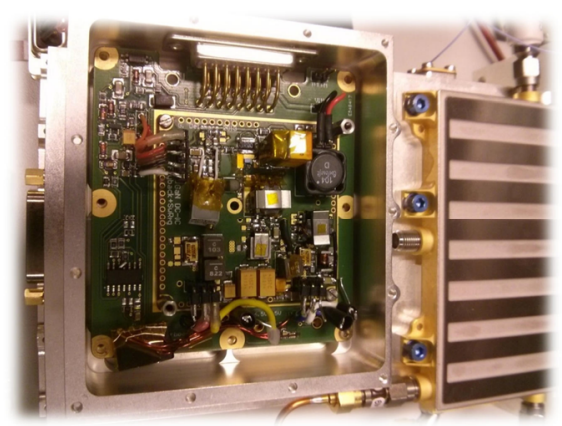

Figure 4. Low power DCDC in RF unit 


\subsection{EPC (Electrical Power Converter) for SSPA}

Then the application on solid state power amplifier was investigated. Usually such power applications uses bridge legs and a specific work has been done on the optimization of legs commutation [1] : through phase opposition method measurements and dedicated software, a prototype has been built and its characteristics was checked in different configurations. Finally, the efficiency was now driven by the losses in the magnetics devices.

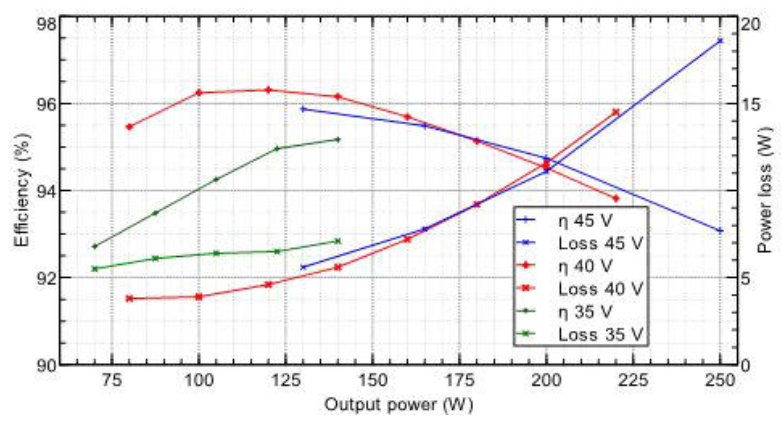

Figure 5. EPC (Electrical Power Converter) for SSPA core efficiency

\subsection{Low voltage application}

GaN transistors were also evaluated to supply digital application needing low voltage $(<3 \mathrm{~V})$ high current $(>10 \mathrm{~A})$ applications. Despite their very low Ron, the conductions losses are still " $\mathrm{I}$ " dependant and parallelisation of several devices has been done owing to intrinsic properties of $\mathrm{GaN}$ device (positive temperature coefficient, ... ).

\section{FEEDBACK \& WAY-FORWARD}

All the above mentioned prototypes allow to get better confidence on the electrical \& thermal aspects of implementing GaN devices in dc-dc converters. On top the central reliability question, there is of course quite a list "space" specific questions that need also to be answered prior to an introduction of $\mathrm{GaN}$ transistors into usual space units.

Hermetic packaging is still needed for most space missions. Hence as specific hermetic packaging needs to be developed for space applications. Currently solutions are converging to SMD05 packages with 4 accesses. The design of this package is a complex exercise to get the heat out of a very small die while minimizing the added parasitic: too high inductance of the connections would completely kill the possibility to increase the switching frequency \& hence kill one of the main interest of using GaN devices. Furthermore this specific process of encapsulating die following standardized ECSS processes will significantly increase the recurrent cost of the component. Today the main factor for selecting a component remains part cost!
Reliability \& Robustness: using components in Space applications requires a complete understanding of the physics of failures. Indeed this allow to design circuits with enough margins (but not too much also) to reach high reliability figures. Over the experiments conducted within Thales several component failures have been observed for a variety of reasons. First one is the high sensitivity of the gate of the GaN. Optimal \& safe gate drive of $\mathrm{GaN}$ is an art on itself.

This raise the question of margins \& deratings to be applied: the low threshold is very low \& can easily be crossed during transients. The performance of the $\mathrm{GaN}$ is only achieved for high gate drive. While doing this the gate voltage come close the max ratings. And there is not a lot of headroom for margins. Crossing the max rating lead to component destruction or highly accelerated aging.

Thales experiments also showed unexpected increases of the gate current. While gate current is negligible on fresh components, silent increase of the leakage current of the gate has been observed both during accelerated aging tests and also during irradiation tests. Of course this change of behavior creates some worries \& we aren't yet able to declare if this would have any impact on the long reliability of the component. These observations all converge to same point: put a lot of attention on the design of gate drivers!

Radiation tests have been conducted also. As published in NASA reports [8], GaN devices are very robust to total ionizing dose. Threshold drift is can almost be considered negligible for most of standard space missions. The breakdown voltage of requires of course requires to define a safe operating area for each transistor when exposed to heavy ions. Finally gate current leakage has been observed and the phenomena is still not yet explained.

\section{CONCLUSION}

Today we are the time of pre-introduction of $\mathrm{GaN}$ HEMT into products. This introduction in products is going its way \& at least isn't completely blocked. Whereas for example $\mathrm{SiC}$ components are still reported to dramatically fail at heavy ion testing.

Promises of higher density thanks to increased switching frequencies are met. Product introduction means also investigating all aspects such as procurement, long term reliability, cooling, radiation robustness. Of course questions \& worries are raising \& they are not fully answered yet, but perspectives are very attractive.

Thales thanks ESA \& CNES teams for their support \& advises during these $\mathrm{GaN}$ investigation projects. 


\section{ABBREVIATIONS AND ACRONYMS}

PPU : Power Propulsion Unit

EPC : Electronic Power Conditioner

EPC-co : Efficient Power Conversion Corp.

\section{REFERENCES}

1. Delamare, G. (2015). GaN HEMT for space power supplies. PhD INP Toulouse.

2. Delamare, G. \& Maynadier, P. (2014). GaN HFET-based DCDC converters for low-power RF satellite equipment, ESPC 2014.

3. Svalgaard, L. \& LeSager, P. (2003). What Heliosesimology Teaches us About the Sun. In Proc. 4th. ISCS 'Solar Variability as an Input to the Earth's Environment' (Eds. H. Lacoste \& H. Xerxes), ESA SP-535 (CD-ROM), European Space Agency, Noordwijk, The Netherlands.

4. GaN survey (Philippe Fayt, Vincent Przybylski, \& Christophe Delepaut) 7th Wide Band Gap Semiconductors and Components Workshop. 1112 September, 2014. Frascati (Rome), Italy

5. Characterization and Application of $600 \mathrm{~V}$ Normally off GaN Transistors in Hard Switching DC/DC Converters (Thomas Heckel and Lothar Frey Stefan Zeltner)

6. Mechanisms responsible for dynamic ONresistance in GaN high-voltage HEMTs Proceedings of the 2012 24th International Symposium on Power Semiconductor Devices and ICs 3-7 June 2012 - Bruges, Belgium Donghyun Jin and Jesús A. del Alamo

7. A very high frequency step-down DC/DC converter for spaceborne envelope-tracking SSPA ( Fabien Leroy · Nicolas Le Gallou · Christophe Delepaut · Olivier Deblecker · Fortunato Dualibe) ESPC-2014

8. Status of the Wide Bandgap Working Group:Gallium Nitride Leif Scheick Jet Propulsion Laboratory, 5th NASA Electronic Parts and Packaging (NEPP) Program Electronic Technology Workshop June 23-26, 2014, NASA GSFC, Greenbelt, MD. 